\title{
Einführung zur deutschen Ausgabe
}

Aus zahlreichen Gründen könnte es heute bei uns einem wissenschaftlich arbeitenden Arzt widerraten erscheinen, durch die Ubersetzung und Herausgabe der deutschen Fassung eines Buches über "Psychosomatischo Medizin" sein intensives Interesse an diesem Stoff zu bekunden. Das Urteil von Physiologen, Internisten, Gynäkologen, Praktikern und weitgehend auch das von Psychiatern, erscheint einstimmig negativ und zum Teil affektiv-betont ablehnend. Jede Parteinahme für diese Forschungsrichtung setzt einen dem Vorwurf aus, statt experimenteller Arbeit mit „Philosophieren“ seine Zeit vergeudet zu haben.

Leider kann man solchen Vorwürfen nicht durchgehend mit selbstsicherem Widerspruch entgegentreten. Die besonderen Bedingungen der Entwicklung der Psychiatrie in unserem Lande, die es mit sich brachten, daß die Psychoanalyse nicht wie in anderen Ländern in das Lehrgebäude der Psychiatrie als grundlegender und integrierender Teil einwuchs und das eigentümlich konservative Verhalten gegenüber der wissenschaftlichen und praktischen Notwendigkeit einer neuen, über die althergebrachte Konsiliartätigkeit hinausgehenden, Form der interdisziplinarischen Zusammenarbeit zwischen den verschiedenen Fachspezialisten haben es mit sich gebracht, daß unter der Bezeichnung Psychosomatik Auffassungen an uns herangetragen worden sind, die auf unseren Unglauben stoßen mußten. Es handelte sich bei uns nicht um Ergebnisse wissenschaftlicher Forschung als einer Gemeinschaftsaufgabe von verbrieften, mit der psychoanalytischen Technik arbeitenden, Psychiatern an Universitäten und Forschungszentren und Fachärzten anderer Disziplinen, die von vornherein per autoritatem mehr Geltung erzwingen müßten. Vielmehr wurde uns das Material vorgestellt von isoliert oder in kleinen Gruppen arbeitenden Psychologen und Psychotherapeuten verschiedener heterogener Schulen, denen es außerdem aus vielerlei Gründen an der notwendigen Kooperation organmedizinisch spezialisierter Mitarbeiter mangelte.

So standen bisher dem Verständnis psychosomatischer Zusammenhänge zwei wesentliche Faktoren entgegen: erstens die Heterogenität der psychologischen Basis der in deutscher Sprache erschienenen Arbeiten, die sich aus der fehlenden Aufnahme der 
Psychoanalyse in das Lehrgebäude der Schulmedizin und den besonderen, nicht-wissenschaftlichen Widerständen in der Entwicklung unserer Tiefenpsychologie ergibt und zweitens die mangelnde Rücksichtnahme der stark geisteswissenschaftlich orientierten Psychotherapeuten auf das für die weitere Forschung doch so unentbehrliche Verständnis des naturwissenschaftlich ausgebildeten Arztes, den sie mit dem Begriff des "Gleichzeitigkeitskorrelates" oder der "Parallelvorgänge im Psychischen“ natürlich nicht näherkommen konnten. Er fühlte bei einer solchen Terminologie mit einigem Recht sein ganzes wissenschaftliches Gebäude, das riesige Erfahrungsgut der Vergangenheit und Gegenwart, bedroht und wehrte sich gegen die Vorstellung, daß man seinem physikochemischen System der Ätiologie von Krankheiten ein gleichwertig umfassendes psychologisches System gegenüberstellen zu können glaubte, das ohne Rücksicht auf die von ihm empirisch und experimentell gesicherten Tatsachen parallel existiere.

So mußte der naturwissenschaftlich denkende Arzt bei uns in der Psychosomatik eine revolutionäre Bewegung erblicken, die wie jede Revolution einen zerstörenden Charakter hat. Jeder echte wissenschaftliche Fortschritt entspricht aber dem Vorgang einer Evolution, wie sie von reiferen Charakteren vorangetrieben wird, die trotz der Neuartigkeit und der umstürzenden Art ihrer Entdeckungen alles vorliegende und geschichtlich gewordene Wissen verbindend einordnen.

Wir glauben allerdings demgegenüber, wenn wir das vorliegende Buch der deutschen Öffentlichkeit präsentieren, eine Revision der mißtrauischen Haltung unserer Leser erreichen zu können, weil in ihm nur systematische, in echter team-Arbeit zwischen Psychiatern und Organmedizinern entstandene Forschungen Aufnahme gefunden haben, und weil es in keinem Falle einer nachgewiesenen psychogenen Beeinflussung körperlicher Vorgänge versäumt worden ist, den direkten Anschluß an die naturwissenschaftlich erforschten und vertrauten physiologischen und pathophysiologischen Tatbestände zu suchen. Es wird uns also hier eine echte „Psychiatrie innerer Krankheiten“ vorgestellt, die direkt kausal an unser gewohntes wissenschaftliches und praktisches Erfahrungsmaterial anschließt. Dieses anschließend Psychologische verlangt seinerseits nicht mehr an Glauben als jede beliebige chemische oder physikalische Technik, die man nicht selbst beherrscht, und deren Ergebnisse daher allein von der Rechtschaffenheit der Untersucher für uns garantiert werden können. Soweit der Blick des Physiologen und des Klinikers reicht, findet sich nichts, was mit seinen Grundideen unverträglich wäre. Es sei denn, er wäre konservativ genug, um zu meinen, daß eine 
chronische funktionelle Störung von genügender Dauer nicht organische Mechanismen so stark belasten und überfordern kann, daß sie ein pathologisch-histologisch faßbares Substrat mit dem Endergebnis einer organisch fixierten Krankheit bedingt.

Wer sich von diesem dogmatischen Einwand befreien kann, der wird hier die an sich selbstverständliche Tatsache erwiesen finden, $\mathrm{da} ß$ neben den rein substantiellen Krankheitsursachen chemischer, bakterieller oder mechanischer Natur auch die keineswegs weniger reale oder weniger bedeutsame menschliche und soziale Umwelt auf dem Wege über unsere Sinnesorgane, ihre intellektuelle und triebmäßige Verarbeitung und die damit verbundenen, endokrin und vegetativ wirksamen, emotionalen Reaktionen zum krankmachenden Agens werden kann. Noch njemand hat aus dogmatischen Gründen einem Pathologen widersprochen, der als Erklärung einer vasomotorischen Berufskrankheit bei Waschfrauen die dauernde funktionelle Uberbeanspruchung der Gefäßmuskulatur der Arme durch den ständigen Wechsel von Kälte- und Wärmereiz behauptet. Ebenso wenig Gründe wird man beim Studium des Buches finden können, um der Beweisführung zu widersprechen, daß die emotionale Verarbeitung der Umweltreize, die ja bei der „,bedingt reflektorischen" Natur zahlreicher primitiver Reaktionsweisen des Lebewesens Mensch meist einen chronisch wiederkehrenden, einseitigen Charakter hat, sozusagen als chronifizierter Affekt, einen wesentlichen ätiologischen Faktor bei vielen sonst ungeklärten „endogenen“ Erkrankungen abgibt.

Freilich verlangt der Psychologe von uns denselben Respekt vor seiner Methodik, den der klinische, bakteriologische oder biochemische Forscher mit Selbstverständlichkeit beansprucht. Nicht unbedingt die oberflächliche, jedem Menschen ohne Spezialstudium zugängliche, Struktur der Psyche grenzt ans Vegetativum, sondern wohl eher die schwerer erschließbaren Anteile der Tiefenpersönlichkeit. Doch sind die zugehörigen Daten in dem Bemühen dargestellt, auch dem nicht psychodynamisch Geschulten das Verständnis zu ermöglichen, wobei doch eine unwissenschaftliche Abflachung vermieden werden konnte. Auf die höhere Mathematik der psychoanalytischen Terminologie ist wo immer angängig verzichtet worden, so daß dem Physiologen, Internisten, Gynäkologen und Praktiker ein Buch vorgelegt werden kann, das neben dem außerordentlichen praktischen Nutzen für seine tägliche Arbeit am Kranken den Weg zu einer forscherischen Zusammenarbeit bei der Aufklärung einer großen Gruppe von Krankheiten eröffnet, der wir erkenntnismäßig und therapeutisch bisher noch immer hilflos gegenüberstehen. Dabei handelt es sich gerade um diejenigen, in ständig steigender Zahl 
jede Praxis überschwemmenden Krankheitsfälle, deren Behandlung die lebenslange Crux jeden Arztes ist, über deren Ätiologie hier Aufklärung zu finden ist und für deren Therapie praktische Wege und neue Aussichten aufgezeigt werden.

Úber diese Nützlichkeitsgesichtspunkte hinaus geht das Buch aber jeden wahren Arzt als solchen an, da es ihm die Berechtigung und Notwendigkeit seines Arzttums, seiner ärztlichen Kunst bestätigt und den schwer erfaßbaren und unkontrollierbaren Faktoren seiner Persönlichkeitswirkung auf seine Kranken faßliche und verwertbare Bereicherungen hinzufügt. Ein begreifbarer pathophysiologischer Mechanismus für eine psychologische Beeinflussung organischer Symptome erst kann uns in unserer Praxis die Kraft der Rechtmäßigkeit beim Einsatz unseres persönlichen Einflusses, zu dessen verständiger und systematischer Anwendung neben dem Medikament und anderen physischen Maßnahmen verleihen.

Manche Psychotherapeuten werden vielleicht enttäuscht sein, wenn sie in diesem Buche keine Details über psychodynamische Behandlungstechniken finden. Der Sinn der Psychosomatik ist jedoch der einer vereinigenden Wissenschaft. Ihre Darstellungen müssen daher primär einen für beide Grenzwissenschaften verständlichen Charakter tragen. Sonst ist ihr Sinn verfehlt. Auch über die Endokrinologie gibt es umfassendere Darstellungen als die hierin enthaltenen. Und doch werden diese ausreichen, um den Machtbereich des psychotherapeutischen Denkens auszudehnen, genau so, wie die psychologischen Forschungsergebnisse dem organmedizinisch geschulten Arzt dienen.

So haben wir, Verleger und Herausgeber, alles in allem doch recht gute Gründe gehabt, das Wagnis der Veröffentlichung dieses Buches über Psychosomatik in deutscherSprache auf uns zu nehmen, weil wir uns überzeugen konnten, daß es diesem Begriff einen wissenschaftlichen Sinn verleiht, der gegen jeden Vorwurf der Spekulation gesichert ist.

Zur Terminologie muß nur gesagt werden, daß wir uns nicht entschließen konnten, die Ausdrücke „emotion“ und „emotional“ in ihre möglichen deutschen Äquivalente "Gefühl, Affekt, psychischer Inhalt" u. dgl. aufzusplittern, sondern sie in ihrer Ganzheit ohne eigentliches Fremdwortgefühl als brauchbar scheinende Termini bestehen gelassen haben. Sie kürzen die Ausdrucksweise und das Denken als systematischer Sammelbegriff für alles Bewegende unter den bewußten und bewußtseinsfähigen psychischen Erscheinungen $\mathrm{ab}$.

Dr. P. Kühne 